\title{
The Impact of Ovarian Endometrioma(s) on ART Outcomes: Retrospective Case Control Study
}

\author{
Emre Göksan PABUÇCU¹, Aslı YARCI GÜRSOY1, Hasan ULUBAŞOĞLU², Özgür Doğuş DEMİRKIRAN, \\ Gamze Sinem ÇAĞLAR ${ }^{1}$, Recai PABUÇCU ${ }^{1,3}$
}

Ankara, Turkey

\begin{abstract}
OBJECTIVE: To compare oocyte yield of women with intact ovarian endometrioma(s) to those without endometrioma undergoing ART. Secondary outcomes were implantation and live birth rates between the two groups.

STUDY DESIGN: Retrospective case-control study was conducted to document eligible cases. A total of 165 women with intact endometrioma(s) (END) were included in the final analysis. Total of 196 cases with tubal factor infertility who underwent ART in the same time period were included as controls (CONT group). Cases and controls were matched for age, BMI and serum AMH concentrations. Ovarian stimulation characteristics and pregnancy outcomes including live birth data were documented for both groups.
\end{abstract}

RESULTS: Despite similar demographic characteristics, significantly longer cycle duration and higher amounts of gonadotropin consumption was observed in END group compared to controls $(p<0.001)$. Significantly lower number of retrieved oocytes, mature oocytes and mature oocyte fraction (\%, no of retrieved/mature) were detected in END group. There was no statistically significant difference in terms of fertilization, implantation and live birth rate per started cycle among groups. Cycle cancellations were also similar.

CONCLUSION: In women with intact ovarian endometrioma(s) undergoing ART, oocyte quantity, especially mature oocyte yield was hampered. However, adequate number of mature oocytes, successful fertilization and satisfactory implantation rates might be possible contributing to acceptable live births. Further randomized controlled trials of patients with different sizes of endometrioma(s) would be needed to confirm our conclusions.

Keywords: Assisted reproductive technologies, Endometrioma, Fertilization, Intracytoplasmic sperm injection, Implantation

Gynecol Obstet Reprod Med 2016;22(2):84-89 DOI: 10.21613/GORM.2016.63

\section{Introduction}

Endometriosis is a serious health burden affecting $0.8-2 \%$ of women at a reproductive age (1). The disease is closely associated with infertility as up to half of infertile women get diagnosed with endometriosis (2,3). A staging system (American Society of Reproductive Medicine Endometriosis Classification) is currently being used to document the severity of the condition and to counsel patients as well (4). Accumulating evidence suggests that advanced stage disease

\footnotetext{
${ }^{1}$ Ufuk University School of Medicine, Department of Obstetrics and Gynecology, Ankara

${ }^{2}$ Kastamonu State Hospital, Department of Obstetrics and Gynecology

${ }^{3}$ Centrum Clinic ART Center, Ankara

Address of Correspondence: Emre Göksan Pabuçcu Ufuk University School of Medicine, Department of Obstetrics and Gynecology Ankara, Turkey pabuccu@hotmail.com

Submitted for Publication 19.03.2016 Accepted for Publication:
}

(III-IV) is associated with poorer reproductive outcomes when compared to earlier stages in women undergoing assisted reproductive technologies (ART) $(5,6)$. Advanced stage endometriosis may exist in several forms and is rather a heterogeneous group. Ovarian endometrioma is another clinical entity that is present in $20-40 \%$ of women with endometriosis (7). Some authors have demonstrated poor ART outcomes in the presence of endometrioma(s) (8); however others have failed to show any detrimental effect on outcomes (9). In this view, infertile women with documented ovarian endometrioma(s) require special attention.

Endometrioma(s) can be identified by transvaginal sonography with high sensitivity and specificity without emerging diagnostic laparoscopy $(10,11)$. Resection of endometrioma(s) prior to ART is controversial. European Society of Human Reproduction and Embryology (ESHRE) guidelines for the diagnosis and treatment of endometriosis recommends laparoscopic surgery before IVF/ICSI in the treatment of symptomatic women with endometrioma and in asymptomatic patients with endometriomas $\geq 4 \mathrm{~cm}$ (12). In the context of dis- 
cussing beneficial effect of surgery, a meta-analysis revealed no significant difference in pregnancy rates or in gonadotropin responses between the surgical management and control groups prior to in-vitro fertilization (IVF) (13). In a more recent Cochrane review, lack of any benefit from either aspiration or cystectomy with regard to clinical pregnancy rates or the number of mature oocytes retrieved was reported when compared to expectant management (14). Besides, accumulating evidence suggests that surgery has a detrimental impact on ovarian reserve in terms of serum Anti-Mullerian hormone $(\mathrm{AMH})$ levels and overall response to ovarian stimulation (OS) (15-17). Accordingly, increasing numbers of authors advocate against surgery prior to ART unless there is refractory pain, significant malignancy potential or inaccessibility to follicles during oocyte retrieval.

As a matter of fact, more and more infertile women with intact ovarian endometrioma(s) are likely to enter an ART programme without having surgery. Hence, there is a need for studies evaluating the noticeable impact of intact ovarian endometrioma(s) on ART outcomes, especially on oocyte yield during ovarian stimulation.

\section{Material and Method}

A chart review of an ART center was performed to detect eligible cases between January 2009 and August 2014. Complete data is comprised of the first intracytoplasmic sperm injection (ICSI) cycle of each couple. All included patients met the following inclusion criteria: subjects between the age of 18 and 40, requiring ART with an indication of primary infertility (women with intact ovarian endometrioma in either ovaries or requiring ART due to tubal factor infertility), women's body mass index (BMI) between 18 and $34 \mathrm{~kg} / \mathrm{m} 2$, thyroid stimulating hormone (TSH) levels $<4,5 \mathrm{IU} / \mathrm{ml}$. Exclusion criteria were: 1) subjects with diminished ovarian reserve, according to the Bologna criteria (18); 2) subjects with documented Mullerian and/or uterine anomaly; 3) GnRH-agonist down regulation longer than 1 month or oral contraceptive use prior to OS; 4) severe oligozoospermia or azoospermia cases; 5) Pre-implantation genetic screening and frozen-thaw embryo transfer cycles; 6) other ovarian cystic appearance rather than endometrioma during transvaginal sonography; 7) women with endometrioma larger than $6 \mathrm{~cm}$ prior to ART. Primary outcome of the study is to compare oocyte yield of women with intact ovarian endometrioma(s) to those without endometrioma undergoing ART. Secondary outcomes were implantation and live birth rates.

During the period, 165 women were detected to have intact ovarian endometrioma(s) while undergoing their first OS cycle without prior surgery (END group). Endometrioma(s) were encountered in the baseline scan for all cases. Ultrasound diagnosis of ovarian endometrioma was based on the visualization of round-shaped homogeneous hypo echoic appearance of low-level echoes within the ovary as previously described (19). The endometrioma was measured in three dimensions, and the average diameter was calculated. Total of 196 cases with tubal factor infertility who underwent ART in the same time period were included as controls (CONT group). Bilateral abnormal tubal patency was documented in all women with hysterosalpingography (HSG) within 3 months of OS. Controls were matched with cases for age, BMI and serum AMH concentrations.

\section{Ovarian Stimulation Protocol}

The study is comprised of one stimulation cycle of each subject in order to prevent possible crossover bias and assignment of subjects to any OS protocol is made by physicians' discretion. Ovarian stimulation was carried out either with GnRH-antagonist (Cetrotide, Merck Serono) (0.25 mg/day) which was initiated when the leading follicle size $>12 \mathrm{~mm}$ during follicular phase or with GnRH-agonist which was administered on the 21 st day of the preceding cycle and then reduced by half when down-regulation was achieved (serum estradiol level $<50 \mathrm{pg} / \mathrm{mL}$ ). Gonadotropin was initiated (hMG (Menogon, Ferring) or recombinant FSH (Gonal-F, Merck Serono)) beginning from the second day of the menstrual cycle with a starting dose of 150-300 IU/day according to the patient's ovarian reserve and body mass index. When at least three follicles were $\geq 18 \mathrm{~mm}$, rhCG $(250 \mu \mathrm{g}$; Ovitrelle, Merck Serono) was used for final oocyte maturation. Transvaginal ultrasound-guided oocyte retrieval and embryo transfer procedure was performed as described elsewhere (20). Manipulations targeting the endometrioma(s) were avoided during the retrieval procedure. One or 2 embryos were transferred. Top quality embryos were defined as those with $\geq 7$ evenly sized cells and $\leq 10 \%$ fragmentation on day 3 and with $\geq 3$ AA quality of blastocyst morphology on day 5 . During the study period, 1 embryo was transferred to patients aged $<35$ years; in patients aged $\geq 35$ years, 2 embryos were transferred, in accordance with local legislation. Clinical pregnancy was defined as the presence of a gestational sac with an embryonic pole and positive heart beat at 7 weeks of gestation and ongoing pregnancy was defined as the presence of an intrauterine sac with an embryonic pole demonstrating cardiac activity at 10 weeks of gestation.

\section{Statistical Analysis}

Data analysis was performed by using SPSS for Windows, version 11.5 (SPSS Inc., Chicago, IL, United States). Whether the distribution of continuous variables was normal or not was determined by Kolmogorov Smirnov test. Data were shown as mean \pm SD or number of cases and (\%). Mean differences between groups were compared by Student's t test whereas Mann Whitney U test was applied for the comparison of median values. Nominal data was analyzed by Pearson's chisquare or Fisher's exact test, where applicable. Correlation analysis was conducted using a Spearman's rank test. A p value less than 0.05 was considered statistically significant. 


\section{Results}

Both groups were compared in terms of OS outcomes, oocyte yield (number of retrieved and mature oocytes), clinical pregnancy rates, live birth rates, cancellations and miscarriages. Cycle cancellations were performed due to lack of ovarian response, fertilization failure or in the presence of no available embryos for transfer.

According to demographic characteristics, mean age, infertility duration, serum AMH levels, basal antral follicle count and TSH levels were similar among groups. Approximately $81 \%$ of subjects in END group had unilateral, whereas $19 \%$ had bilateral endometriomas. The median diameter of ovarian endometrioma was $3(1-6) \mathrm{cm}$. Total progressive motile sperm count (sperm concentration/ml X volume (ml) X motility (+4 fraction)) of the groups was statistically similar ( $p>0,05)$. In END group, 79\% (130/165) of subjects underwent OS with agonist and 21\% (35/165) of them underwent with antagonist protocol. Demographic characteristics of groups are shown in Table 1.

Significantly higher amount of gonadotropin $(2978 \pm 1135$ vs. $2022 \pm 749 \mathrm{IU})$ and higher cycle duration (11,2 vs. 9,9 days) was detected in END group $(p<0,001)$. Significantly lower number of retrieved oocytes (6.5 \pm 2.6 vs. $9.0 \pm 5.7)$, mature oocytes (3.5 \pm 2.5 vs. $6.7 \pm 4.2)$ and mature oocyte fraction $\%$ (no of retrieved/mature) $(53.7 \%$ vs. $78.5 \%)$ were detected in END group, when compared to controls ( $p<0,001)$. There was no statistical difference in terms of implantation, ongoing pregnancy rate/started cycle (OPR) and live birth rate/started cycle (LBR) among groups. Cycle cancellations were also similar between groups. Cycle characteristics and pregnancy outcomes of groups are shown in Table 2 and in Table 3. In END group, OPR (22,3\% with antagonist, $14,3 \%$ with agonist; $p=0,298)$ and LBR $(21,5 \%$ with antagonist, $14,3 \%$ with agonist; $p=0,341$ ) were similar in both OS protocols.

According to endometrioma localization, there were similar mature oocytes obtained either in subjects with bilateral or with unilateral endometriomas $(2.9 \pm 2.7$ vs. $3.6 \pm 2.5$ respectively, $\mathrm{p}=0,176)$. Relatively higher OPR (6,4\% vs. $19,4 \%$, $\mathrm{p}=0,083)$ and LBRs $(6,4 \%$ vs. $17,9 \%, \mathrm{p}=0,170)$ were detected in subjects with unilateral disease, however the difference did not reach statistical significance.

Table 1: Demographic characteristics of the groups

\begin{tabular}{lccc}
\hline & $\begin{array}{c}\text { END Group } \\
(\mathrm{n}: 165)\end{array}$ & $\begin{array}{c}\text { Control Group } \\
(\mathrm{n}: 196)\end{array}$ & $\mathrm{p}$ value \\
\hline Age (years) & $34.33 \pm 5.36$ & $33.24 \pm 5.25$ & 0.068 \\
Duration of marriage (years) & $5(1-16)$ & $7(1-14)$ & 0.053 \\
AMH (ng/mL) & $2.50(1-7)$ & $3(1-5)$ & 0.081 \\
AFC $(\mathrm{n})$ & $9.32 \pm 3.37$ & $9.64 \pm 3.02$ & 0.354 \\
TSH (mU/L) & $1.80(0.45-4.5)$ & $1.82(0.25-4.5)$ & 0.423 \\
TPMSC (x106) & $50(9-234)$ & $45(10-230)$ & 0.572 \\
Unilateral endometrioma, $\mathrm{n}(\%)$ & $134 / 165(81.3)$ & - & - \\
Bilateral endometrioma, $\mathrm{n}(\%)$ & $31 / 165(18.7)$ & & \\
Endometrioma size $(\mathrm{cm})$ & $3(1-6)$ & - & \\
\end{tabular}

Values are expressed as mean \pm standard deviation, *Values are expressed as median with minimum value and maximum value parenthesis. Statistically significant $p$-values are in bold.

AMH: Anti mullerian hormone, AFC: Antral follicle count, TSH: Thyroid stimulating hormone, TPMSC: Total progressive motile sperm count

Table 2: Ovarian stimulation outcomes of the groups

\begin{tabular}{lccr}
\hline & $\begin{array}{c}\text { END Group } \\
(\mathrm{n}: 165)\end{array}$ & $\begin{array}{c}\text { Control Group } \\
(\mathrm{n}: 196)\end{array}$ & $\mathrm{p}$ value \\
\hline Total dose $(\mathrm{IU})$ & $2.978 .58 \pm 1135.69$ & $2.022 .90 \pm 749.23$ & $<0.001$ \\
E2 on hCG day $(\mathrm{pg} / \mathrm{mL})$ & $1.690(5-5625)$ & $1.905 .50(72-5670)$ & 0.051 \\
Endometrial thickness $(\mathrm{mm})$ & $10.25 \pm 1.93$ & $10.70 \pm 2.19$ & 0.054 \\
Total duration (days) & $11.24 \pm 2.12$ & $9.99 \pm 2.93$ & $<0.001$ \\
Number of retrieved oocytes $(\mathrm{n})$ & $6.54 \pm 2.64$ & $9.06 \pm 5.75$ & $<0.001$ \\
Mature (MII) (n) & $3.50 \pm 2.59$ & $6.76 \pm 4.28$ & $<0.001$ \\
MII \% & $53.7 \pm 29.55$ & $78.54 \pm 24.85$ & $<0.001$ \\
Fertilization \% & $61.86 \pm 33.78$ & $68.06 \pm 29.65$ & 0.067 \\
\hline
\end{tabular}

Values are expressed as mean \pm standard deviation, *Values are expressed as median with minimum value and maximum value parenthesis. Statistically significant $p$-values are in bold.

E2: Estradiol, MII: Mature 
Table 3: Ovarian stimulation and pregnancy outcomes of the groups

\begin{tabular}{lccc}
\hline & $\begin{array}{c}\text { END Group } \\
(\mathrm{n}: 165)\end{array}$ & $\begin{array}{c}\text { Control Group } \\
(\mathrm{n}: 196)\end{array}$ & $\mathrm{p}$ value \\
\hline Implantation rate & $16.61 \pm 33.77$ & $20.75 \pm 35.05$ & 0.255 \\
Clinical pregnancy rate, $\mathrm{n}(\%)$ & $35(21.2)$ & $59(30.1)$ & 0.055 \\
Ongoing pregnancy rate, $\mathrm{n}(\%)$ & $34(20.6)$ & $56(28.6)$ & 0.081 \\
Live Birth, $\mathrm{n}(\%)$ & $33(20.0)$ & $51(26.0)$ & 0.177 \\
Miscarriages, $\mathrm{n}(\%)$ & $2(1.2)$ & $16(8.6)$ & 0.063 \\
Cycle cancellation, $\mathrm{n}(\%)$ & $17(10.3)$ & & 0.482 \\
\hline
\end{tabular}

Values are expressed as mean \pm standard deviation

According to Spearman's analysis, negative correlation was detected between endometrioma size and oocyte yield both in terms of total number of retrieved $(r=-0.129$, $\mathrm{p}=0.103)$ and mature oocytes $(\mathrm{p}=-0.116, \mathrm{p}=0.142)$, where differences are not statistically significant.

During OS, there were 4 mild pelvic inflammatory diseases encountered which was associated with the oocyte retrieval procedure. All cases were managed in the outpatient clinic with oral antibiotics without any complication. There were no septic conditions detected including pelvic abscess requiring hospitalization during the study period.

\section{Discussion}

Based on the findings of the current study, women with intact endometrioma(s) are likely to reveal lower number of oocytes during OS resulting with decreased number of mature oocytes. This relatively low ovarian response is observed even with higher gonadotropin consumption and longer duration. However, if obtained, fertilization and implantation rates were not compromised when compared to controls. Accordingly, live birth rates were not hampered despite lower oocyte yield in the presence of endometrioma(s).

To date, few studies have specifically examined the ART outcomes of women with intact endometrioma(s) regardless of stage. In those studies, endometrioma cases were compared to that of other infertility etiologies including male or tubal factor infertility (8,21-23). A recent two meta-analysis have focused on the OS characteristics and ART outcomes of women with intact endometriomas $(6,24)$. According to Hamdan et al, women with intact endometrioma revealed lower number of retrieved oocytes when compared to women with no endometriosis (SMD -0.23; 95\% CI (-0.37, -0.10) (6). Similarly, pooled data from the meta-analysis of Yang et al revealed 1.50 fewer oocytes retrieved in women with ovarian endometrioma than in those without endometrioma (WMD 1.50 ; $95 \%$ CI -2.84 to $-0.15, \mathrm{P}=0.03$ ) (24). Authors also provided information about embryos, as they suggested a reduction in good-quality forms in patients with endometrioma. In our study, significantly lower oocytes were harvested in women with endometrioma despite higher gonadotropin usage and longer duration. Mature oocyte fraction was also found to decrease in END group. Interestingly, in our study and in the meta-analyses, implantation and live birth rates were found to be similar among those with or without endometriomas. Such results allow speculation that the ovarian endometriosis per se exerts some detrimental impact on the oocyte quantity rather than quality. In our data, mature oocyte number was lower in END group, but fertilization and implantation rates were similar. This might be suggestive of a theory as existence of endometrioma may compromise the oocyte quantity rather than fertilization or implantation capacity. If we could obtain adequate number of oocytes from the ovary with endometrioma, successful implantation can be achieved. Supportively, it has recently been shown that the presence of endometrioma does not compromise time-lapse morphokinetics of embryos (25). Decreased oocyte quantity might be due to some molecular detrimental interactions caused by cytokines, mechanical distortion of ovarian cortex or by oxidative stress contributing to relatively decreased ovarian response, mainly induced by the presence of endometrioma discussed previously (24). These mechanisms may contribute to decreased ovarian response, but in the availability of enough mature oocyte, fertilization and implantation capacity seems not to be compromised, resulting with live births. Similar fertilization, implantation and live birth rates either in our study or in the above mentioned meta-analyses further indicate that the oocyte quality seems not to be hampered by the endometrioma.

In women undergoing ART, the optimal management of endometriomas is still debated. According to the ESHRE guidelines, laparoscopic surgery before IVF/ICSI is considered to be the 'gold standard' in the treatment of symptomatic endometriomas (12). On the other hand, surgery indeed has a detrimental impact on ovarian reserve in terms of serum AMH levels and overall response to OS (15-17), despite enough surgical experience (26-28). In this context, size of the endometrioma seems to be the crucial determinant in management. Esinler et al. demonstrated that the unilateral endometriomas $\leq 3 \mathrm{~cm}$ in diameter did not have a deleterious effect on ovarian reserve in ICSI cycles (29). Recently, Cocia et al. observed a critical endometrioma size of $3 \mathrm{~cm}$, in those with unilateral cyst, above this size, which the total numbers of follicles and retrieved oocytes were negatively affected 
(26). For every millimeter of increase in endometrioma size, the predicted number of retrieved oocytes was shown to decrease by 0,667 . We documented significantly lower oocyte yield with the mean endometrioma diameter of $3.2 \mathrm{~cm}( \pm 1.2$ SD) that is consisted with of findings of Cocia et al. Those in END group had mature oocytes which were one half as low as those of a control group. Negative correlation was also documented between endometrioma size and oocyte yield, in terms of total number of retrieved and mature oocytes, however this difference was not statistically significant all of the above studies have evaluated unilateral cases and compared the overall response with that of contralateral ovaries. In our study, not all but $81 \%$ of endometriomas were unilateral. Nevertheless, we did not evaluate the response of contralateral ovary in each subject, as this is the limitation of the current study. Size limit of $3 \mathrm{~cm}$, particularly in those with a unilateral cyst should be taken into account even deciding for conservative management or surgery. This finding seems to be more important especially in those with reduced ovarian reserve.

Obviously, there is a need for studies evaluating the efficacy of different OS protocols particularly for this special population. Administration of GnRH agonists for a period of three to six months prior to ART has been shown to increase pregnancy odds (30). However, this analysis was limited only to 3 studies without specifically examining those with intact endometrioma(s). There is currently no solid evidence to support a routine use of a particular OS protocol, despite few studies that have revealed similar pregnancy outcomes either with agonists or antagonists $(31,32)$. In our study, both OS protocols yielded comparable OPR and LBRs in END group without any significant difference. However, randomized and adequately powered studies are necessary to drive a conclusion.

Moreover, in the case of a conservative approach, the risk of an ovarian abscess after ovarian puncture during ultrasound-guided oocyte retrieval should be taken into account. According to Somigliana et al. (33), several drawbacks including septic complications, technical difficulties or occult malignancy risk might be associated with the conservative approach. Late pregnancy complications following aspiration of endometrioma have also been reported in the literature (34). In our study, we did not observe severe complications except few mild pelvic infections, however long term careful follow-up is necessary for women, particularly when consecutive ART cycles are decided. On the potential benefits and harms of both conservative management and surgery to share the consequences of decision.

In conclusion, oocyte quantity was hampered during OS when there is ovarian endometrioma(s). However, if OS results with adequate number of mature oocytes, successful fertilization following an ICSI and satisfactory implantation rates might be possible contributing factors to acceptable live births. Further randomized controlled trials of patients with different sizes of endometriomas would be needed to confirm our conclusions.

\section{References}

1. Leibson CL, Good AE, Hass SL, Ransom J, Yawn BP, O'Fallon WM et al. Incidence and characterization of diagnosed endometriosis in a geographically defined population. Fertil Steril 2004;82:314-21

2. Ozkan S, Murk W, Arici A. Endometriosis and infertility: epidemiology and evidence-based treatments. Ann N Y Acad Sci 2008;1127:92-100

3. Houston DE, Noller KL, Melton LJ III, Selwyn BJ, Hardy RJ. Incidence of pelvic endometriosis in Rochester, Minnesota, 1970-1979. Am J Epidemiol 1987;125:959-69

4. Revised American Fertility Society classification of endometriosis: 1985. Fertil Steril 1985;43:351-2

5. Harb HM, Gallos ID, Chu J, Harb M, Coomarasamy A. The effect of endometriosis on in vitro fertilization outcome: a systematic review and meta-analysis. BJOG 2013; 120:1308-20

6. Hamdan M, Omar SZ, Dunselman G, Cheong Y. Influence of endometriosis on assisted reproductive technology outcomes: a systematic review and meta-analysis. Obstet Gynecol 2015;125:79-88

7. Vercellini P, Chapron C, De Giorgi O, Consonni D, Frontino G, Crosignani PG. Coagulation or excision of ovarian endometriomas? Am J Obstet Gynecol 2003;188: 606-10

8. Yanushpolsky EH, Best CL, Jackson KV, Clarke RN, Barbieri RL, Hornstein MD. Effects of endometriomas on oocyte quality, embryo quality, and pregnancy rates in in vitro fertilization cycles: a prospective, case-controlled study. J Assist Reprod Genet 1998;15:193-7

9. Hamdan M, Dunselman G, Li TC, Cheong Y. The impact of endometrioma on IVF/ICSI outcomes: a systematic review and meta-analysis. Hum Reprod Update 2015;21(6): 809-25

10. Alcazar JL, Laparte C, Jurado M, Lopez-Garcia G. The role of transvaginal ultrasonography combined with color velocity imaging and pulsed Doppler in the diagnosis of endometrioma. Fertil Steril 1997;67:487-91

11. Eskenazi B, Warner M, Bonsignore L, Olive D, Samuels S, Vercellini P. Validation study of nonsurgical diagnosis of endometriosis. Fertil Steril 2001;76:929-35

12. Kennedy S, Bergqvist A, Chapron C, D'Hooghe T, Dunselman G, Greb R et al. ESHRE Special Interest Group for Endometriosis and Endometrium Guideline Development Group, 2005. ESHRE guideline for the diagnosis and treatment of endometriosis. Hum Reprod $2005 ; 20: 2698-2704$

13. Tsoumpou I, Kyrgiou M, Gelbaya TA, Nardo LG. The effect of surgical treatment for endometrioma on in vitro fer- 
tilization outcomes: a systematic review and meta-analysis. Fertil Steril 2009;92(1):75-87

14. Benschop L, Farquhar C, van der Poel N, Heineman MJ. Interventions for women with endometrioma prior to assisted reproductive technology. Cochrane Database Syst Rev 2010;(11):CD008571

15. Raffi F, Metwally M, Amer S. The impact of excision of ovarian endometrioma on ovarian reserve: a systematic review and meta-analysis. J Clin Endocrinol Metabol 2012; 97:3146-54

16. Somigliana E, Berlanda N, Benaglia L, Vigano $\mathrm{P}$, Vercellini P, Fedele L. Surgical excision of endometriomas and ovarian reserve: a systematic review on serum antimullerian hormone level modifications. Fertil Steril 2012;98:1531-8

17. Muzii L, Di Tucci C, Di Feliciantonio M, Marchetti C, Perniola G, Panici PB. The effect of surgery for endometrioma on ovarian reserve evaluated by antral follicle count: a systematic review and meta-analysis. Human Reprod 2014;29:2190-8

18. Ferraretti AP, La Marca A, Fauser BC, Tarlatzis B, Nargund G, Gianaroli L. ESHRE consensus on the definition of 'poor response' to ovarian stimulation for in vitro fertilization: the Bologna criteria. Hum Reprod 2011; 26:1616-24

19. Moore J, Copley S, Morris J, Lindsell D, Golding S, Kennedy S. A systematic review of the accuracy of ultrasound in the diagnosis of endometriosis. Ultrasound Obstet Gynecol 2002;20:630-4

20. Pabuccu EG, Caglar GS, Pabuccu R. Estrogen or anti-estrogen for Bologna poor responders? Gynecol Endocrinol 2015;31:955-8

21. Ashrafi M, Fakheri T, Kiani K, Sadeghi M, Akhoond MR. Impact of the endometrioma on ovarian response and pregnancy rate in in vitro fertilization cycles. Int $\mathrm{J}$ Fertil Steril 2014;8:29-34

22. Suzuki T, Izumi S, Matsubayashi H, Awaji H, Yoshikata $\mathrm{K}$, Makino T. Impact of ovarian endometrioma on oocytes and pregnancy outcome in in vitro fertilization. Fertil Steril 2005;83:908-13

23. Bongioanni F, Revelli A, Gennarelli G, Guidetti D, Delle Piane LD, Holte J. Ovarian endometriomas and IVF: a retrospective case-control study. Reprod Biol Endocrinol 2011;9:81

24. Yang C, Geng Y, Li Y, Chen C, Gao Y. Impact of ovarian endometrioma on ovarian responsiveness and IVF: a systematic review and meta-analysis. Reprod Biomed Online 2015;31(1):9-19

25. Demirel C, Bastu E, Aydogdu S, Donmez E, Benli H, Tuysuz $\mathrm{G}$ et al. The Presence of Endometrioma Does Not Impair Time-Lapse Morphokinetic Parameters and Quality of Embryos: A Study On Sibling Oocytes. Reprod Sci 2016 Feb 10.

26. Coccia ME, Rizzello F, Barone S, Pinelli S, Rapalini E, Parri $\mathrm{C}$ et al. Is there a critical endometrioma size associated with reduced ovarian responsiveness in assisted reproduction techniques? Reprod Biomed Online 2014;29 (2):259-66

27. de Ziegler D, Borghese B, Chapron C. Endometriosis and infertility: pathophysiology and management. Lancet 2010;376:730-8

28. Garcia-Velasco JA, Somigliana EM. Management of endometriomas in women requiring IVF: to touch or not to touch. Hum Reprod 2009;24:496-501

29. Esinler I, Bozdag G, Arikan I, Demir B, Yarali H. Endometrioma $\leq 3 \mathrm{~cm}$ in diameter per se does not affect ovarian reserve in intracytoplasmic sperm injection cycles. Gynecol Obstet Invest 2012;74(4):261-4

30. Sallam HN, Garcia-Velasco JA, Dias S, Arici A. Longterm pituitary down-regulation before in vitro fertilization (IVF) for women with endometriosis. Cochrane Database Syst Rev 2006;CD004635

31. Pabuccu R, Onalan G, Kaya C. GnRH agonist and antagonist protocols for stage I-II endometriosis and endometrioma in IVF/ ICSI cycles. Fertil Steril 2007;88: $832-9$

32. Rodriguez-Purata J, Coroleu B, Tur R, Carrasco B, Rodriguez I, Barri PN. Endometriosis and IVF: are agonists really better? Analysis of 1180 cycles with the propensity score matching. Gynecol Endocrinol 2013;29: 859-62

33. Somigliana E, Benaglia L, Paffoni A, Busnelli A, Vigano P, Vercellini P. Risks of conservative management in women with ovarian endometriomas undergoing IVF. Hum Reprod Update 2015;21(4):486-99

34. Okyay E, Özay AC, Emekci Ö, Bodur ART, Doğan ÖE. A case of pregnancy complicated by the development of a Tubo-ovarian abscess following an aspiration of endometrioma during Oocyte pick up. Gynecol Obstet Reprod Med 2014;20(3):172-4 